\title{
Modelagem da distribuição de frequência de teores carbono orgânico do solo sob Floresta Ombrófila Mista no Paraná
}

\author{
Tauane Garcia Barreto ${ }^{1}$, Ângela Maria Klein Hentz ${ }^{1}$, Sylvio Péllico Netto ${ }^{1}$, Ana Paula Dalla Corte ${ }^{1}$, Carlos Roberto Sanquetta ${ }^{1}$ \\ 1 Universidade Federal do Paraná, Av. Pref. Lothário Meissner, 900, Jardim Botânico, Campus III, CEP 80210-170, Curitiba, PR, Brasil
}

*Autor correspondente:

tauanebiologia@gmail.com

Termos para indexação:

Função densidade de probabilidade

Função Gama

Matéria orgânica do solo

Index terms:

Probability density function

Gamma function

Soil organic matter

Histórico do artigo:

Recebido em 18/05/2015

Aprovado em 17/03/2017

Publicado em 31/03/2017

doi: 10.4336/2017.pfb.37.89.953

\begin{abstract}
Resumo - Neste trabalho objetivou-se modelar o teor de carbono no solo de parcelas sob Floresta Ombrófila Mista em condições distintas (predomínio de araucária, de imbuia e vegetação mista com registro de ocorrência de fogo), no Paraná, a partir de distribuições probabilísticas. Para tal, foi avaliada a qualidade de ajuste e aderência de diferentes funções de probabilidade (normal, Weber, Weibull 3P, log-normal, SB de Johnson e Gama), pelo teste de Kolmogorov-Smirnov. Todas as funções densidade de probabilidade resultaram em bons ajustes, podendo então ser utilizadas para modelar os dados de carbono no solo em qualquer uma das parcelas. As funções foram ranqueadas a partir dos valores do teste Kolmogorov-Smirnov, e decidiu-se que a função Gama é a mais apropriada para modelar o teor de carbono nas três parcelas estudadas. Ainda, ao realizar a comparação das distribuições Gama ajustadas pelo teste Kolmogorov-Smirnov nas diferentes condições testadas, observou-se que elas apresentam configurações distintas, portanto, devem ser modeladas individualmente.
\end{abstract}

\section{Modeling of the frequency distribution of soil organic carbon of Araucaria Forest in Paraná State}

\begin{abstract}
This work aimed to describe the frequency distribution of soil organic carbon contents under different conditions of Araucaria forest (concentration of araucaria trees, imbuia trees and mixed forest, with record of fire) in Paraná State, Brazil. Fitness quality of probability density functions (normal, Weber, Weibull 3P, log-normal, SB of Johnson and Gamma) were determined, using Kolmogorov Smirnov test. All functions fitted adequate, so they can all be used for any tested condition. Functions were ranked using Kolmogorov-Smirnov test. Gamma function was selected as the most appropriate for all tested conditions. However, after a comparison of fitted distributions by KolmogorovSmirnov test, we concluded that modeling must be performed separately.
\end{abstract}

\section{Introdução}

A definição de estatística mais utilizada atualmente descreve que essa é uma área do conhecimento que utiliza teorias probabilísticas para explicação de eventos, estudos e experimentos, com o objetivo de obter, organizar e analisar dados, determinar e interpretar correlações para descrição, explicação e previsão (Weber, 2006). Dentro desse contexto, podem ser utilizadas as distribuições probabilísticas, que permitem prever fenômenos a partir de modelos matemáticos, tornando possível o estudo do comportamento de variáveis do meio físico ou biológico, denominadas funções densidade de probabilidade (Silva et al., 2003).

As funções probabilísticas contínuas, também conhecidas como funções densidade de probabilidade, podem ser agrupadas quanto aos picos de frequência (unimodal ou multimodais), simetria (simétricas ou assimétricas), tipo de curvatura (rígida ou flexível) e número de variáveis aleatórias (univariadas ou 
multivariadas) (Guimarães, 2002). Segundo Padovani (2012), pode-se caracterizar uma variável aleatória contínua como uma mensuração cujos possíveis valores ocorrem aleatoriamente e pertencem a um intervalo dos números reais.

Todavia, a utilização de funções de distribuição de probabilidade requer o uso de testes para provar a adaptação dos dados às funções, conhecidos como testes de aderência, os quais visam verificar a forma de uma distribuição, através da análise da adequação dos dados à curva de um modelo de distribuição hipotética (Araújo et al., 2010). Dentre outros testes de aderência, pode-se citar Kolmogorov-Smirnov, que se baseia na diferença entre a frequência acumulada estimada com a observada (Leite et al., 2010), da qual obtém-se um valor máximo que é comparado com um limite tabelado.

Atualmente, na ciência florestal tem-se produzido diversos trabalhos utilizando as funções densidade de probabilidade, principalmente no que diz respeito à modelagem de crescimento e produção, embora estudos com a mesma temática tenham sido pouco explorados na área de solos florestais. Dentre esses poucos estudos sobre solos florestais, podem-se citar Lima et al. (2003), que objetivaram agrupar parte das espécies arbóreas de uma floresta primária do Estado do Amapá, Amazônia Oriental, por meio das características do solo. Os autores concluíram que a distribuição normal se ajustou satisfatoriamente para as concentrações de vários nutrientes do solo, incluindo o carbono, e algumas características edáficas.

Outros estudos sobre solos e teores de carbono são encontrados em áreas não florestais. McGrath \& Zhang (2003) utilizaram a distribuição de probabilidade normal para modelar a concentração de carbono orgânico no solo sob pastagem na Irlanda. Estes autores observaram que a distribuição dos dados logaritmizados adere a essa distribuição no caso do carbono orgânico, enquanto que outras variáveis, como teor de argila, aderem a essa distribuição sem necessidade de transformações.

A importância dos estudos de carbono em área florestais está no grande poder de neutralização e a estocagem do carbono que as florestas apresentam (Ribeiro et al., 2005), principalmente nos solos florestais. Estes, segundo Machado (2005), apresentam estoques de carbono superiores aos demais ecossistemas terrestres, como por exemplo nos encontrados na biomassa vegetal ou na atmosfera.
Contudo, a manutenção dos estoques de carbono depende, dentre outros fatores, do manejo aplicado ao solo, como observado por Matias et al. (2012), e, portanto, é importante considerar uma proposta metodológica que integre o mapeamento, o inventário florestal, as determinações de biomassa, as determinações dos teores de carbono e a modelagem matemática para a quantificação e o monitoramento de carbono (Sanquetta et al., 2006). Desse modo, o objetivo deste trabalho foi modelar estatisticamente a distribuição dos teores de carbono no solo sob Floresta Ombrófila Mista, no Município de São João do Triunfo, PR, por meio das funções densidade de probabilidade e, adicionalmente, observar como se comportam as distribuições em área com predomínio de árvores de araucária, de imbuia e em área com vegetação mista com registro de presença de fogo.

\section{Material e métodos}

O estudo foi executado na Estação Experimental da Universidade Federal do Paraná, localizada no município de São João do Triunfo, PR. Possui uma área total de aproximadamente 32 ha, fragmentada em 30 parcelas permanentes, tendo como coordenadas geográficas

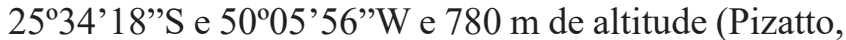
1999).

Trata-se de um fragmento de Floresta Ombrófila Mista, também chamada de Floresta com Araucária, o qual, segundo Pizatto (1999), apresenta estágio sucessional aparentemente diferente. Os solos dessa região são associações de Argissolos Vermelho-Amarelo, Cambissolos Háplicos Tb e Latossolos Vermelhos, predominantemente distróficos (Durigan, 1999). Os dados da composição química das parcelas estudadas encontram-se na Tabela 1.

De acordo com Barreto (2015), os solos das parcelas estudadas apresentam condição de elevada acidez, alta concentração de alumínio $\left(\mathrm{Al}^{+3}\right)$ e baixa saturação por bases, indicando locais de baixa fertilidade natural. Quanto aos aspectos físicos do solo, Maas (2015) em estudos na mesma área, reportou textura predominantemente argilosa, com teores médios de argila, silte e areia de $45,7 \%, 25,1 \%$ e $29,1 \%$ respectivamente, em profundidade de $0-20 \mathrm{~cm}$. 
Tabela 1. Composição química do solo nas parcelas de estudo, em fragmento de Floresta Ombrófila Mista, localizado em São João do Triunfo, PR.

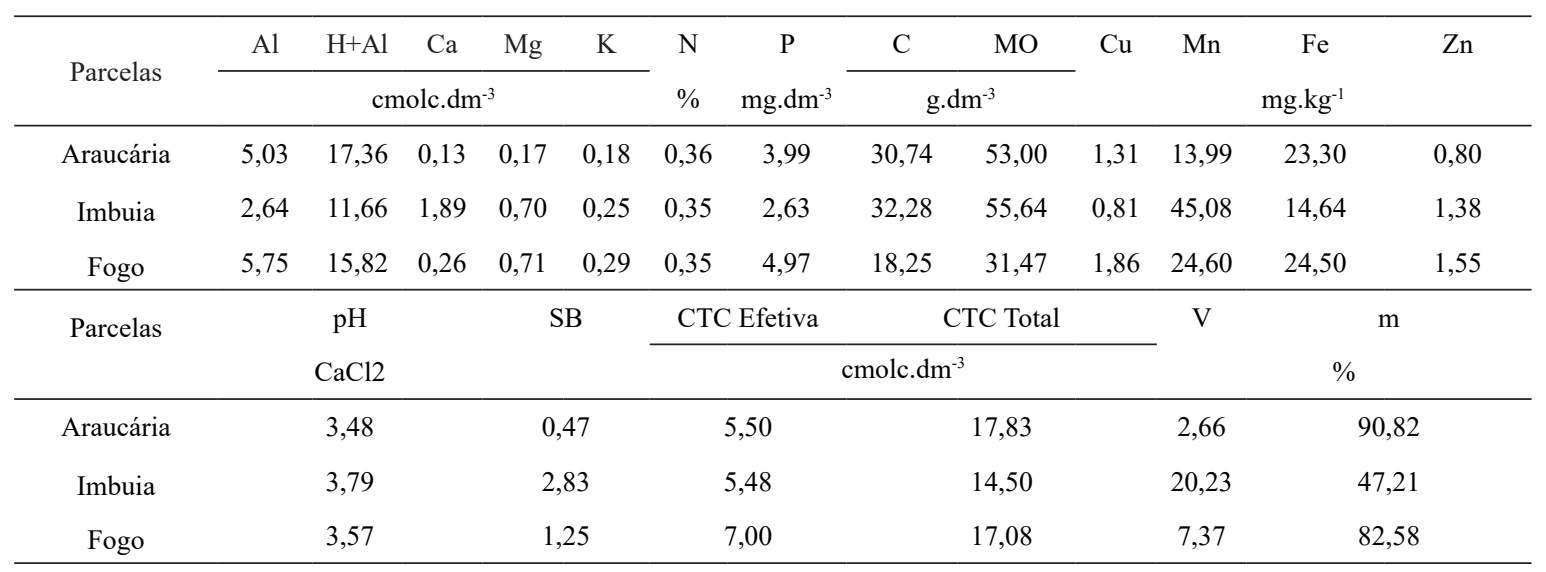

Nota: $\mathrm{SB}=$ soma de bases (\%); CTC efetiva $=$ capacidade de troca cationnica efetiva (cmolc.dm-3); CTC total = Capacidade de troca catiônica total (cmolc.dm-3); V = saturação por bases (\%); $\mathrm{m}$ = saturação por alumínio (\%); MO = matéria orgânica (\%). Fonte: Barreto (2015)

O clima da região é do tipo $\mathrm{Cfb}$, segundo a classificação climática de Köppen, temperado e úmido, com a temperatura média anual oscilando entre 17 e $18{ }^{\circ} \mathrm{C}$, umidade relativa anual entre 75 e $85 \%$ e precipitação anual média variando entre 1.400 e $1.600 \mathrm{~mm}$ (Caviglione et al., 2000).

Foram selecionadas três parcelas para este estudo, com 1 ha cada, sendo nomeadas como araucária (parcela com predominância de Araucaria angustifolia (Bertol.) Kuntze no dossel), fogo (parcela mista com Araucaria angustifolia (Bertol.) Kuntze e espécies folhosas no dossel, que no passado foi atingida por um incêndio) e imbuia (parcela com predominância de espécies folhosas no dossel, especialmente Ocotea porosa (Nees \& C.Mart.) Barroso).

As três parcelas permanentes foram subdivididas em sub-parcelas, medindo $10 \mathrm{~m}$ x $10 \mathrm{~m}$ cada, totalizando 100 subparcelas em cada parcela. A cada 4 sub-parcelas foi retirada uma amostra de solo, totalizando 25 amostras por parcela. Para compor uma amostra foram coletadas três amostras simples, localizadas de forma diagonal (duas nos extremos da área amostral, e uma no centro), formando uma amostra completa. A tradagem foi realizada com trado Holandês, na profundidade de $0-20 \mathrm{~cm}$.
As amostras de solo foram secas em estufa a $42{ }^{\circ} \mathrm{C}$, maceradas, peneiradas com malha de $2 \mathrm{~mm}$, pesadas com balança analítica de precisão e identificadas. Posteriormente, a análise de carbono foi realizada por combustão a seco e detecção por espectroscopia de infravermelho, com analisador de carbono LECO SC144, resultando nos teores de carbono para cada amostra em porcentagem.

Com base nos teores de carbono obtidos, foram ajustadas seis diferentes funções de densidade de probabilidade (Tabela 2), visando identificar a melhor para descrever os valores de carbono nas três parcelas. Cada parcela dispunha de 25 amostras, consideradas suficientes pelo cálculo do número amostral ideal com base no coeficiente de variação.

Os ajustes foram avaliados pelo teste de aderência de Kolmogorov-Smirnov, ao nível de 95\% de probabilidade, no qual as frequências cumulativas dos dados observados são comparadas aos das distribuições teóricas. Além disso, foi realizado um ranqueamento (pesos) dos ajustes, atribuindo-se o valor um à função com melhor ajuste e valores crescentes para as outras funções, conforme sua colocação em ordem de qualidade de ajuste, sendo o melhor ajuste aquele com valor mais distante do valor tabelado do teste de KolmogorovSmirnov, possibilitando avaliar qual distribuição foi mais adequada, a partir da soma de pesos em todas as parcelas. 
Tabela 2. Distribuições ajustadas e testadas para os dados de carbono no solo.

\begin{tabular}{|c|c|c|}
\hline Função & Expressão matemática & Condicionantes \\
\hline Normal & $f(x)=\frac{1}{\sigma \sqrt{2 \pi}} e^{-\frac{(x-\mu)^{2}}{2 \sigma^{2}}}$ & $\begin{aligned} \sigma & >0 \\
-\infty & <x<+\infty \\
-\infty & <\mu<+\infty\end{aligned}$ \\
\hline Weber & $f(x)=\frac{x^{a}}{(b+c x)^{d}}$ & $\begin{array}{c}0 \leq x<+\infty \\
d \geq a+1 \\
d \neq a+2 \\
d \neq a+3 \\
a, b, c, d>0\end{array}$ \\
\hline Weibull 3P & $f(x)=\frac{\gamma}{\beta}\left(\frac{x-\alpha}{\beta}\right)^{\gamma-1} \exp \left[-\left(\frac{x-\alpha}{\beta}\right)\right]^{\gamma}$ & $\begin{array}{c}x \geq x_{\min } \\
-\infty<x_{\min } \\
<+\infty \\
b, c>0 \\
a \geq 0\end{array}$ \\
\hline Log-normal & $f(x)=\frac{1}{x \sigma \sqrt{2 \pi}} \exp \left[-\frac{(\log x-)^{2}}{2 \sigma^{2}}\right]$ & $\begin{array}{c}x \geq x_{\min } \\
\sigma>0 \\
-\infty<x<+\infty \\
-\infty<\mu<\infty\end{array}$ \\
\hline $\mathrm{S}_{\mathrm{B}}$ de Johnson & $f(x)=\frac{\delta}{2 \pi} * \frac{\lambda}{x-\varepsilon \lambda+\varepsilon-x} * \exp ^{\left(-\frac{1}{2} \gamma+\delta * \ln \frac{x-\varepsilon}{\lambda+\varepsilon-x}\right)^{2}}$ & $\begin{array}{c}\varepsilon<x<+\lambda \\
-\infty<\gamma<+\infty \\
\gamma>0 \\
\delta>0\end{array}$ \\
\hline Gama & $f(x)=\frac{1}{\beta^{\alpha} \Gamma(\alpha)} x^{\alpha-1} e^{-\frac{x}{\beta}}$ & $\begin{array}{c}x \geq x_{\min } \\
\propto, \beta>0 \\
-\infty<x_{\min } \\
<+\infty\end{array}$ \\
\hline
\end{tabular}

fx: função de densidade probabilística da variável x; $\mathrm{x}$ : variável aleatória; $\mu$ : média dos valores de $\mathrm{x}$; $\sigma$ : desvio padrão de x; xmin: valor mínimo de x; xmáx : valor máximo de x; $\pi$ : 3,14159...; e: constante de Euler; a, b, c, d, $\alpha, \beta, \gamma, \delta, \varepsilon, \lambda$ : parâmetros a serem estimados.

\section{Resultados e discussão}

Os teores de carbono no solo, em porcentagem, bem como seus parâmetros estatísticos estão apresentados na Tabela 3.

A parcela araucária apresentou a maior média de carbono (4,66\%). Maciel (2012), em estudos realizados em fragmento florestal com predominância de Anadenanthera sp. em Minas Gerais, na profundidade de 0-20 cm, obteve teor de carbono médio inferior (3,09\%). Da mesma forma, outros valores de teor de carbono em áreas de Mata Atlântica são apresentados por Inácio (2009) que observou valor médio de teor de carbono entre $0-20 \mathrm{~cm}$, de $3,24 \%$ em área com mata nativa, assim como $2,5 \%$ de valor médio até $20 \mathrm{~cm}$ de profundidade encontrado por Rangel \& Silva (2007).
Tabela 3. Estatística descritiva para os teores de carbono no solo nas parcelas de estudo.

\begin{tabular}{cccc}
\hline \multirow{2}{*}{ Estatísticas } & \multicolumn{3}{c}{ Teor de Carbono (\%) } \\
& Araucária & Fogo & Imbuia \\
\hline Média & $4,66 \mathrm{a}$ & $3,81 \mathrm{~b}$ & $3,56 \mathrm{~b}$ \\
Mínimo & 3,60 & 2,96 & 2,12 \\
Máximo & 6,04 & 4,70 & 6,18 \\
Mediana & 4,61 & 3,82 & 3,28 \\
Variância da & 0,34 & 0,20 & 0,91 \\
amostra & 0,59 & 0,45 & 0,95 \\
Desvio padrão & 0,15 & $-0,31$ & 1,63 \\
Curtose & 0,38 & $-0,10$ & 1,17 \\
Assimetria &
\end{tabular}

Valores de média seguidos pela mesma letra não apresentam diferença significativa pelo teste de Tukey, ao nível de $95 \%$ de probabilidade. Teste de Levene para homogeneidade das variâncias igual a 2,62, $\mathrm{p}=0,079$. 
Valores mais próximos ao da área com concentração de imbuias e onde houve registro de fogo foi encontrado por Sandi (2009), que relatou média de 3,7\% até 20 $\mathrm{cm}$. Para Floresta Ombrófila Mista é destacado o valor de cerca de $8 \%$ encontrado por Baretta (2007), com profundidade de $0-20 \mathrm{~cm}$.

É notório que existe uma grande variabilidade de carbono no solo, pela análise da literatura disponível e pelos dados obtidos nesse trabalho. Isso já era esperado, visto que, conforme Gatto et al. (2010), a capacidade de fixação e estocagem de carbono no solo depende de fatores como condições climáticas locais, relevo, drenagem, tipo de manejo de solo adotado, tipo de cobertura vegetal, profundidade do solo, entre outras. Quanto a estes fatores, a comparação entre florestas nativas e plantios florestais comumente é levantada, e é apresentada por Fialho \& Zinn (2014), que observaram diversos estudos sobre perda de carbono no solo em função da alteração da cobertura florestal original por plantios de eucalipto. Fialho \& Zinn (2014) observaram que as perdas de $\mathrm{C}$ apresentam distribuições de frequências tendendo à normalidade, tanto para as profundidades de $0-20 \mathrm{~cm}$ como $0-40 \mathrm{~cm}$, de forma que as perdas, em sua maior parte, são próximas à zero.

De acordo com Caldeira et al. (2002) e Mafra et al. (2008), a adição de material orgânico proveniente, principalmente da serapilheira, é responsável pelo acúmulo de carbono na camada superficial do solo, à medida que essa vai sendo humificada. Logo, pode-se inferir que a superioridade em teor de carbono orgânico no solo da parcela Araucária ocorreu por conta da representatividade em número de indivíduos da espécie Araucaria angustifolia (Bertol.) Kuntze em relação às demais parcelas. Segundo Maas (2015), essa espécie contribui não apenas com a maior parte do estoque de carbono na biomassa, mas também seus ramos aciculados são a fração mais representativa no retorno de carbono ao piso florestal via serapilheira.

O desvio padrão bem como a variância apresentaram valores considerados baixos, de forma que os dados tendem a estar menos dispersos e mais próximos do valor da média, embora a parcela imbuia tenha se apresentado divergente, com os maiores valores para essas estatísticas, comparado às demais parcelas.

Apenas a parcela fogo apresentou assimetria negativa, pois a média encontrada foi menor que a mediana dos dados, ou seja, existem mais amostras com valores situados acima do valor médio. Ressalta-se, porém, que as parcelas fogo e araucária têm baixíssimas assimetrias, ou seja, tendem a uma distribuição normal, enquanto que a parcela imbuia apresenta maior disformidade em relação a uma distribuição normal. A distribuição dos dados de carbono nas parcelas fogo e araucária apresentou tendência semelhante, leptocúrtica (curtose $<0,263$ ), enquanto que os dados na parcela imbuia apresentaram distribuição platicúrtica (curtose $>0,263$ ).

Observa-se uma tendência de comportamentos que poderiam modelar a distribuição mais adequada. Contudo, Mesquita et al. (2003) ressaltam que, apesar disto, esses valores não informam sobre o tipo de distribuição, que permitiria determinar além da média e da dispersão, o número de amostras a serem coletadas e a probabilidade que um dado valor assumiria dentro de limites especificados.

$\mathrm{O}$ ajuste das funções densidade de probabilidade, bem como a representação das curvas de frequência, sob o gráfico de valores observados estão apresentados na Figura 1.

A partir dos resultados dos ajustes de dados pelo teste de Kolmogorov-Smirnov, (Tabela 4), foi possível constatar que todas as distribuições testadas se ajustaram aos dados para as três parcelas analisadas. A aderência nesse teste é dada pela aceitação da hipótese nula $\left(\mathrm{H}_{0}\right)$, que nesse caso é de que a distribuição dos dados segue a distribuição teórica em análise.

Assis et al. (2013) ressaltam que além do ajuste das funções, existe a necessidade do uso de critérios e testes de aderência para verificar se a distribuição de probabilidade dos dados pode ser representada por uma determinada função de distribuição probabilística conhecida. Assis et al. (1996) apontam que os testes aderência de qui-quadrado e Kolmogorov-Smirnov são amplamente utilizados, porém, em geral, o teste de Kolmogorov-Smirnov é mais rigoroso (Costa Neto, 2002). Segundo Campos (1979), o teste KomogorovSmirnov pode ser aplicado, sem restrições, para pequenas amostras, além de possibilitar o tratamento dos dados de forma individual, evitando assim a perda de informações por agrupamentos, o que justifica o seu uso nesse estudo.

O ranqueamento a partir dos valores do teste de Kolmogorov-Smirnov está apresentado na Tabela 5. 
Parcela Araucária

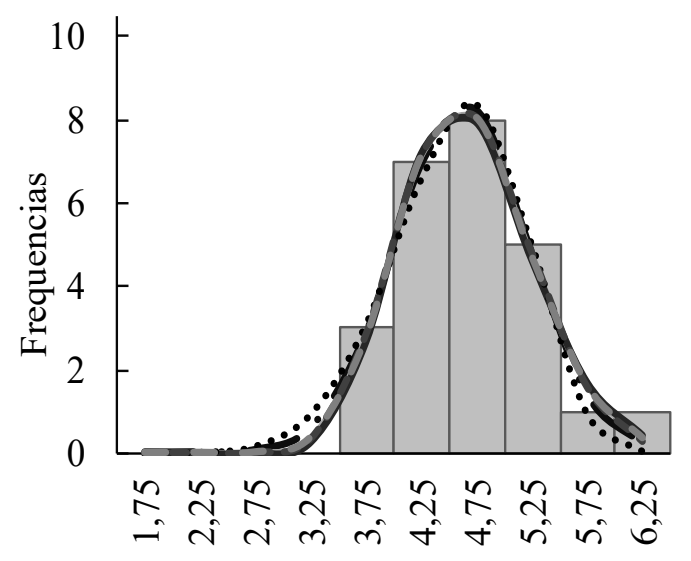

Teores de carbono no solo (\%)

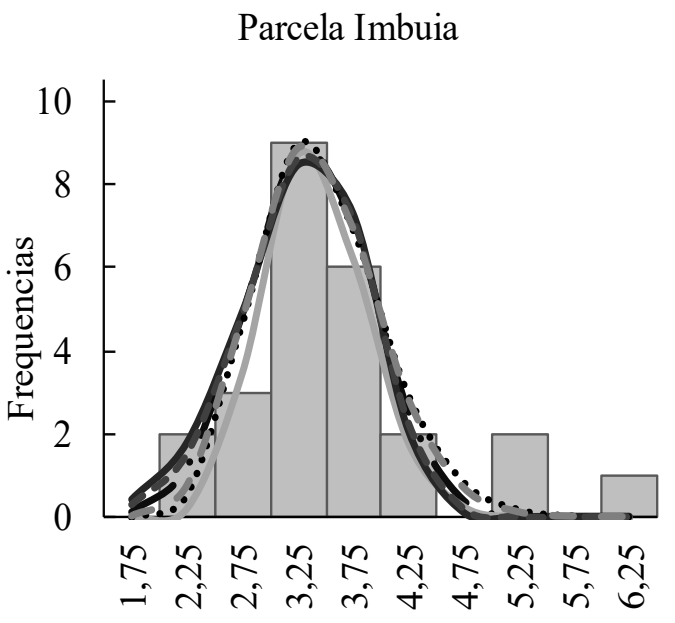

Teores de carbono no solo (\%)

\section{Parcela Fogo}
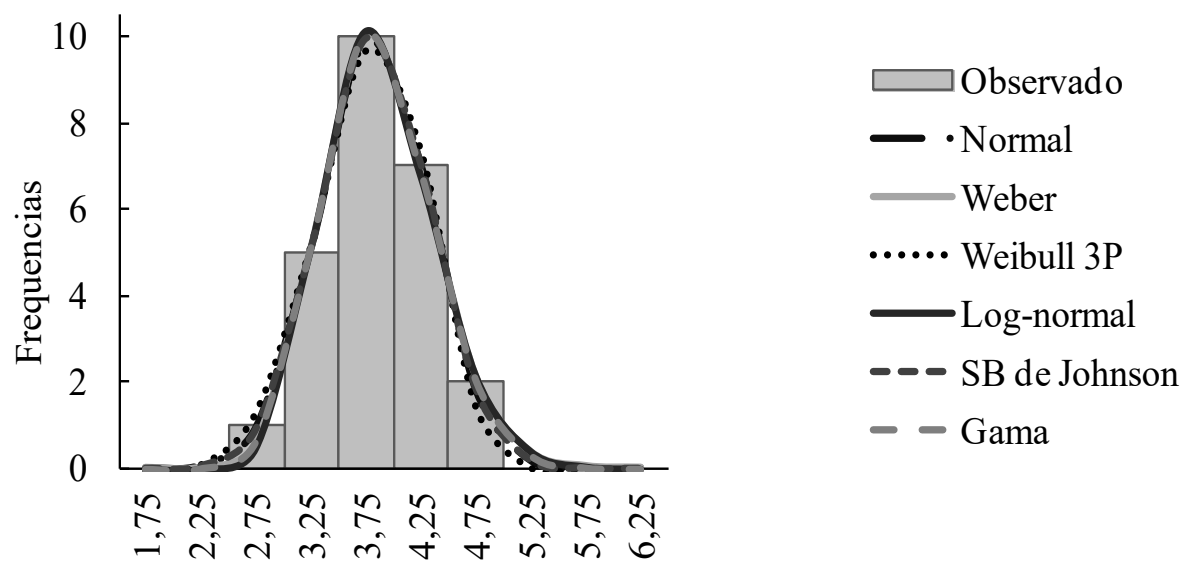

Teores de carbono no solo (\%)

Figura 1. Ajustes das distribuições em comparação com os valores observados de carbono no solo.

Tabela 4. Resultado dos ajustes pelo teste de KolmogorovSmirnov para os teores de carbono no solo.

\begin{tabular}{cccc}
\hline \multirow{2}{*}{ Funções } & \multicolumn{3}{c}{ Kolmogorov-Smirnov } \\
\cline { 2 - 4 } & Araucária & Fogo & Imbuia \\
\hline Normal & $0,0317 \mathrm{~ns}$ & $0,0071 \mathrm{~ns}$ & $0,1185 \mathrm{~ns}$ \\
Weber & $0,0246 \mathrm{~ns}$ & $0,0180 \mathrm{~ns}$ & $0,1693 \mathrm{~ns}$ \\
Weibull 3P & $0,0458 \mathrm{~ns}$ & $0,0203 \mathrm{~ns}$ & $0,1181 \mathrm{~ns}$ \\
Log-normal & $0,0180 \mathrm{~ns}$ & $0,0254 \mathrm{~ns}$ & $0,1109 \mathrm{~ns}$ \\
SB de Johnson & $0,0284 \mathrm{~ns}$ & $0,0078 \mathrm{~ns}$ & $0,1181 \mathrm{~ns}$ \\
Gama & $0,0236 \mathrm{~ns}$ & $0,0169 \mathrm{~ns}$ & $0,1147 \mathrm{~ns}$ \\
\hline $\mathrm{ns}=$ não significativo, para 95\% de probabilidade
\end{tabular}

$\overline{\text { ns }}=$ não significativo, para $95 \%$ de probabilidade.
Tabela 5. Ranqueamento da qualidade dos ajustes das distribuições, pelos parâmetros do teste de KolmogorovSmirnov.

\begin{tabular}{ccccccc}
\hline \multirow{2}{*}{ Parcela } & \multicolumn{5}{c}{ Ranqueamento - Kolmogorov-Smirnov } \\
\cline { 2 - 7 } & Normal & Weber & $\begin{array}{c}\text { Weibull } \\
\text { 3P }\end{array}$ & Log-normal & $\begin{array}{c}\text { SB de } \\
\text { Johnson }\end{array}$ & Gama \\
\hline Araucária & 5 & 3 & 6 & 1 & 4 & 2 \\
Fogo & 1 & 4 & 5 & 6 & 2 & 3 \\
Imbuia & 5 & 6 & 3 & 1 & 4 & 2 \\
Soma & 11 & 13 & 14 & 8 & 10 & 7 \\
\hline
\end{tabular}

Nessa comparação, a distribuição Gama resultou no melhor ajuste para os dados, visto que a soma do ranqueamento desta apresentou menor valor. Esta 
et al. (2007) encontraram excelentes resultados para a distribuição Gama em análises de nutrientes dentro de partições de árvores, como boro, por exemplo.

A distribuição log-normal ficou em segundo lugar no ranqueamento, e apresentou-se como a melhor distribuição para as parcelas araucária e imbuia, apesar de ser o pior ajuste obtido para a parcela fogo. A aplicação da função log-normal para modelagem de atributos do solo também foi utilizada por Pereira et al. (2014) para modelar a probabilidade de falhas geológicas resultantes das alterações nas propriedades rochosas devido ao processo conhecido como captura e sequestro de carbono, feito de forma artificial por injeção em rochas profundas que, segundo os autores, pode causar a reativação de falhas geológicas. Esses autores apontam que a teoria das probabilidades é uma ferramenta poderosa para alcançar um bom equilíbrio entre as consequências de conhecimento incerto e o custo da redução dessa incerteza.

Os parâmetros da distribuição Gama (Tabela 6) foram próximos para as parcelas araucária e fogo, porém distintos para a parcela imbuia. No entanto, observa-se que mesmo pequenas variações nos coeficientes podem proporcionar distribuições com formatos distintos.

Tabela 6. Parâmetros da distribuição Gama para as três parcelas.

\begin{tabular}{ccc}
\hline Parcela & $\boldsymbol{\alpha}$ & $\boldsymbol{\beta}$ \\
\hline Araucária & 60,04586 & 0,07758 \\
Fogo & 60,10376 & 0,06431 \\
Imbuia & 37,13273 & 0,09235 \\
\hline
\end{tabular}

O comportamento da quantificação dos teores de carbono no solo para as parcelas modeladas pela distribuição Gama está apresentado na Figura 2. É possível observar que as três curvas se comportam de maneira distinta, principalmente na localização das áreas de maior frequência. Também se verifica que os valores de carbono aumentam substancialmente da parcela imbuia para fogo e atinge o máximo na parcela araucária.

Para confirmar a existência de diferença entre as curvas, aplicou-se o teste de Kolmogorov-Smirnov entre os valores estimados para cada parcela pela função Gama. Observou-se que todas as curvas diferem entre si, a um nível de 95\% de probabilidade. Dessa forma, é natural supor que as três parcelas apresentam configurações distintas, as quais afetam os teores de carbono disponíveis no solo sob vegetação.

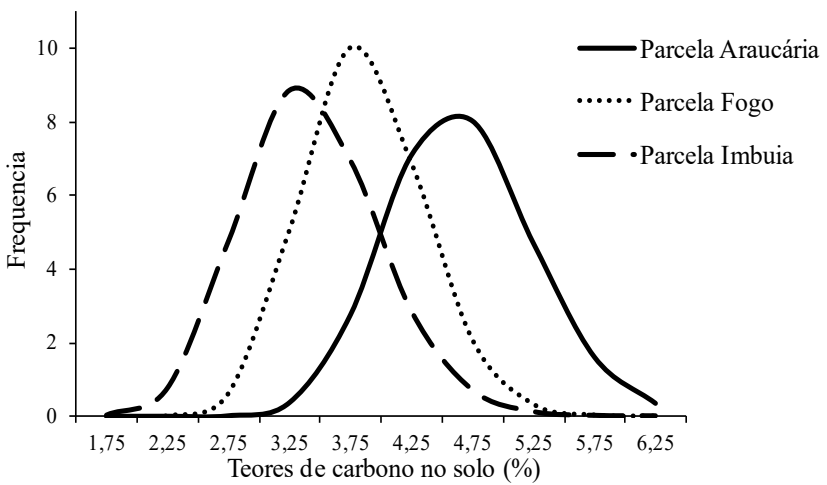

Figura 2. Quantidade de carbono modelado pela distribuição Gama para as parcelas.

\section{Conclusões}

Os dados de carbono no solo nas condições em estudo de Floresta Ombrófila Mista, com concentração de araucária, concentração de imbuia e em área com registro de fogo, podem ser modelados por qualquer uma das funções de probabilidade testadas, visto que todas apresentaram aderência às distribuições dos dados das parcelas. Apesar disto, destaca-se que a distribuição log-normal é a mais adequada para as parcelas araucária e imbuia, enquanto que distribuição normal é a mais adequada para a parcela fogo. A função Gama é a mais indicada no caso em que se queira utilizar uma única função para as três condições, pois ela apresentou um bom desempenho no ajuste das três parcelas.

Ao aplicar-se o teste de Kolmogorov-Smirnov entre as parcelas, considerando para todas a distribuição Gama, observou-se que não houve aderência entre elas, ou seja, as parcelas apresentam distribuições distintas, mesmo quando utilizada a mesma função. Dessa forma, apesar de ser possível utilizar uma única função, o ajuste para cada parcela deve ser realizado individualmente.

\section{Referências}

Araújo, E. M. et al. Aplicação de seis distribuições de probabilidade a séries de temperatura máxima em Iguatu - CE. Revista Ciência Agronômica, v. 41, n. 1 p. 36-45, 2010.

Assis, F. N. et al. Aplicações de estatística à climatologia: teoria e prática. Pelotas: UFPEL, 1996. 161 p.

Assis, J. P. et al. Ajuste de sete modelos de distribuições densidade de probabilidade às séries históricas de umidade relativa mensal em Mossoró - RN. Revista Verde, v. 8, n. 1, p. 1-10, 2013. 
Barreto, T. G. Geoestatística aplicada à modelagem da dinâmica de crescimento e características químicas do solo de um fragmento florestal. 2015. 187 f. Dissertação (Mestrado em Engenharia Florestal) - Universidade Federal do Paraná, Curitiba.

Baretta, D. Fauna do solo e outros atributos edáficos como indicadores da qualidade ambiental em áreas com Araucaria Angustifolia no Estado de São Paulo. 2007. 158 f. Tese (Doutorado em Agronomia) - Escola Superior de Agricultura Luiz de Queiroz, São Paulo.

Caldeira, M. V. W. et al. Carbono orgânico em solos florestais. In: Sanquetta, C. R. et al. (Ed.). As florestas e o carbono. Curitiba: UFPR, 2002. p. 191-213.

Campos, H. Estatística experimental não-paramétrica. 4. ed. Piracicaba: Departamento de Matemática e Estatística, ESALQ, 1979. 349 p.

Caviglione, J. H. et al. Cartas climáticas do Paraná. Londrina: IAPAR, 2000. Disponível em: <http://www.iapar.br/modules/ conteudo/conteudo.php?conteudo=677>. Acesso em: 06 jun. 2015.

Costa Neto, P. L de O. Estatística. 2. ed. São Paulo: Edgar Blucher, 2002. 280 p.

Dallacort, R. et al. Distribuição das chuvas no município de Tangará da Serra, médio norte do Estado de Mato Grosso, Brasil. Acta Scientiarum Agronomy, v. 33, n. 2, p. 193-200, 2011. DOI: 10.4025/ actasciagron.v33i2.5838.

Durigan, M. E. Florística, dinâmica e análise proteica de uma Floresta Ombrófila Mista em São João do Triunfo - PR. 1999. 125 f. Dissertação (Mestrado em Ciências Florestais) - Universidade Federal do Paraná, Curitiba.

Fialho, R. C. \& Zinn, Y. L. Changes in soil organic carbon under Eucalyptus plantations in Brazil: a comparative analysis. Land Degradation \& Development, v. 25, p. 428-437, 2014. DOI: 10.1002/ldr.2158.

Gatto, A. et al. Estoques de carbono no solo e na biomassa em plantações de eucalipto. Revista Brasileira de Ciência do Solo, v. 34, n. 4, p. 1069-1080, 2010.

Guimarães, D. P. Uma função hiperbólica de distribuição probabilística de alta flexibilidade. Planaltina, DF: Embrapa Cerrados, 2002. 40 p. (Embrapa Cerrados. Documentos, 79).

Inácio, E. S. B. Distribuição vertical de carbono orgânico em Latossolo sob diferentes usos. 2009. 86 f. Tese (Doutorado em Ciências do Solo) - Universidade Federal de Lavras, Lavras.

Leite, H. G. et al. Avaliação do ajuste das funções Weibull e hiperbólica a dados de povoamentos de eucalipto submetidos a desbaste. Revista Árvore, v. 34, n. 2, p. 305-311, 2010. DOI: 10.1590/S0100-67622010000200013.

Lima, J. A. S. et al Agrupamento de espécies arbóreas de uma floresta tropical por características de solo. Pesquisa Agropecuária Brasileira, v. 38, n. 1, p. 109-116, 2003. DOI: 10.1590/S0100204X2003000100015.

Lúcio, A. D. et al. Distribuição de probabilidade em análises nutricionais de espécies florestais. Revista Ceres, v. 54, n. 313, p. 215-225, 2007.
Maas, G. C. B. Compartimentação do estoque de carbono em Floresta Ombrófila Mista. 2015. 172 f. Tese (Doutorado em Engenharia Florestal) -Universidade Federal do Paraná, Curitiba.

Machado, P. L. O. A. Carbono do solo e a mitigação da mudança climática global. Química Nova, v. 28, n. 2, p. 329-334, 2005. DOI: 10.1590/S0100-40422005000200026.

Maciel, S. M. Análise espacial do carbono em um fragmento florestal com predominância de Anadenanthera sp. 2012. 134 f. Dissertação (Mestrado em Ciência e Tecnologia da Madeira) Universidade Federal de Lavras, Lavras.

Mafra, A. L. et al. Carbono orgânico e atributos químicos do solo em áreas florestais. Revista Árvore, v. 32, n. 2, p. 217-224, 2008. DOI: $10.1590 / \mathrm{S} 0100-67622008000200004$.

Matias, S. S. R. et al. Influência de diferentes sistemas de cultivo nos atributos físicos e no carbono orgânico do solo. Revisa Brasileira de Ciência Agrária, v. 7, n. 3, p. 414-420, 2012. DOI: 10.5039/ agraria.v7i3a1462.

McGrath, D. \& Zhang, C. S. Spatial distribution of soil organic carbon concentrations in grassland of Ireland. Applied Geochemistry, v. 18, p. 1629-1639, 2003. DOI: 10.1016/S0883-2927(03)00045-3.

Mesquita, M. G. B. F. et al. Caracterização estatística de variáveis físicas do solo. Acta Scientiarum Agronomy, v. 25, n. 1, p. 35-44, 2003. DOI: 10.4025/actasciagron.v25i1.2342.

Padovani, C. R. Bioestatística. São Paulo: Cultura Acadêmica, Universidade Estadual Paulista, 2012. 113 p.

Paiva Sobrinho, S. de et al. Determinação dos parâmetros da distribuição gama e média pluviométrica decendial para estações do estado de Mato Grosso. Revista Brasileira de Meteorologia, v. 29, n. 2, p. 183-196, 2014. DOI: 10.1590/S0102-77862014000200004.

Pereira, F. L. G. et al. Fault reactivation case study for probabilistic assessment of carbon dioxide sequestration. International Journal of Rock Mechanics \& Mining Sciences, 71, p. 310-319, 2014. DOI: 10.1016/j.ijrmms.2014.08.003.

Pizatto, W. Avaliação biométrica da estrutura e da dinâmica de uma Floresta Ombrófila Mista em São João do Triunfo - PR: 1995 a 1998. 1999. 172 f. Dissertação (Mestrado em Engenharia Florestal) - Universidade Federal do Paraná, Curitiba.

Rangel O. J. P. \& Silva C. A. Estoques de carbono e nitrogênio e frações orgânicas de Latossolo submetido a diferentes sistemas de uso e manejo. Revista Brasileira de Ciência do Solo, v. 31: p. 1609-1623, 2007. DOI: 10.1590/S0100-06832007000600037.

Ribeiro S. C. et al. Quantificação de biomassa e estimativa de estoque de carbono em uma floresta madura no município de Viçosa, Minas Gerais. Revista Árvore, v. 33, n. 5, p. 917-927, 2005. DOI: 10.1590/ S0100-67622009000500014.

Sandi, J. T. T. Estoque de carbono no solo sob diferentes coberturas vegetais, Chapecó-SC. 2009. 83 f. Dissertação (Mestrado em Ciências Ambientais) - Universidade Comunitária Regional de Chapecó, Chapecó.

Sanquetta, C. R. et al. Proposta metodológica para quantificação e monitoramento do carbono estocado em florestas plantadas. In: Sanquetta, C. R. et al. (Ed.). Carbono: desenvolvimento tecnológico, aplicação e mercado global. Curitiba, 2006. p. 240-265. 
Silva, E. Q. de et al. Função densidade de probabilidade aplicável à ciência florestal. Revista Floresta, v. 33, n. 3, p. 285-294, 2003.

Soccol, O. J. et al. Análise da precipitação mensal provável para o município de Lages, SC. Revista Brasileira de Engenharia Agrícola e Ambiental, v. 14, p. 569574, 2010. DOI: 10.1590/S141543662010000600001 .
Weber, S. H. Desenvolvimento de nova função densidade de probabilidade para avaliação de regeneração natural. 2006. 74 f. Dissertação (Mestrado em Engenharia Florestal) - Universidade Federal do Paraná, Curitiba. 
